\title{
Hybrid Approach of Spectrum-Energy Efficiency Optimization for LTE Network
}

\author{
Y.D. Chincholkar ${ }^{1}$, Mohammed Luay Abdulmunem ${ }^{2}$ \\ E\&TC Dept., Sinhgad College of Engineering, Pune (MH), India ${ }^{1,2}$
}

\begin{abstract}
A massive research is growing across the spectrum efficiently in networks such as LTE, based on 802.22 standards. Along with the spectrum efficiency by the mobile phone service point of view, the important energy savings is nothing but the reduction in operating costs and improve the efficiency of the spectrum is nothing more than increase profits. To improve profitability, it is necessary that the wide area network (wireless access networks) has efficient energy and spectrum. The division of this work are designed to offer a new hybrid approach, aimed at improving energy efficiency and spectrum Advance LTE cellular networks. We have a new policy for allocating optimal planning methods proposed size spectrum swelling, migration of users and distribution of various types of base stations sleep mode. A problem spectrum efficiency and energy optimization method is calculated as quasiconvex and then transforming a problem MILP. The optimization problem is solved quasiconvex bisection method and solved by a software tool MILP. Finally, we have contributed to the third algorithm to improve spectrum efficiency and energy in this paper. The proposed method practical simulation work performed using NS2. A practical simulation results show that the efficiency of the method is better than previous methods.
\end{abstract}

Keywords: Energy, Spectrum, LTE, Heterogeneous networks, Base station, Cellular, 802.22.

\section{INTRODUCTION}

The development of communication systems has greatly increased, we are hoping to use essential tools to improve the capacity and throughput for the 4th generation. With this type of technical force to strengthen mobile phone operators, information services are receiving an annual increase in traffic volume data. Meanwhile, more than $18 \%$ of operating expenses come from the base station (BSS), $60 \%$ of the network equipment and energy consumption occupying the bill's point. The amount of information expands to improve the benefits of using expenses for expansions of vitality. From the viewpoint of portable server, I showed interest in keeping cost-effective in wireless network [1] [2]. It was analyzed deploying different types of BSs is able to help on increase revenues and minimize expenditure. Thus a great margin of maneuver for the network operators makes it possible to obtain financial improvements in the hybrid cellular deployment. HetNets is one concern about the increase in the number of local cell numbers, so energy-efficient operation in heterogeneous environments is an important issue in order to reduce energy consumption hand, the impact on spectrum efficiency should also be taken into account, when calculating the energy saving mechanisms, [4].

From the literature studies, Energy efficiency and energy efficiency performance have been shown to complement each other as energy efficiency decreases the efficiency of the spectrum. Plans for joint enhancement of phantom productivity and vitality should be considered as many. However, these two changes in productivity problems of the system are covered and centered on the other side in writing. Therefore, in this paper, for heterogeneous network environments, it has been developed model of spectral energy efficiency optimization. They define the energy spectrum efficiency in the network by increasing the optimization of the spectrum distribution while reducing the total energy consumption [8] [9]. It thus becomes the longest research problem of energy spectrum research. The spectrum efficiency spectrum can be considered as a measure to help indicate the viability of operators, quantify the revenue capacity for costs. It is an objective function that aims to increase the proportion of the range in relation to the devoured vitality skill for the administrative task [10]. The goal is to create a system plan that is the percentage of information for controlled and responsible businesses, which is progressing. Since an effective system is adapted to increase the volume of information, it is necessary to take an effective way, with regard to the use of vitality at a given cost, the income is then constructed, derived from Amplify Published information. With the ideal rate for asset management, can be calculated the most advantageous configuration of the system.

\section{LITERATURE REVIEW}

We have presented a study on the latest effective spectrum techniques for LTE or CR networks.

In [1], the author introduces an approach where the Cognitive Radio Hubs are interested in choosing the accessibility of the range. The playback result shows that linear and polynomial classifiers give a high key customer recognition rate 
with constant false alarm rate in terms of cutting traumatic barriers. In all cases, the techniques proposed can reach over $90 \%$ of the probability of discovery $\mathrm{Eb} / \mathrm{No}=-7 \mathrm{~dB}$ with $10 \%$ false alarms and the 50-bit perception window. As the detection time of both techniques increases, it is proven that the performance in advance.

In [2], the author presented the proposed channel usage forecast model, based on user mobility and Cognitive Radio Spectrum Transmission. The expected use of the time chain is a key measure for sharing the spectrum. In order to reduce channel transfers resulting in short time usage channel, a usage threshold is set for channel assignment. When the transfer channel cannot be implemented in a single hop, multi-hop routing will be established to maintain communication.

In [3], the author of this article, taking directions from our previous execution of the work of algorithms based on instantaneous and simple CUSUM DE for single-node spectrum detection, said algorithm of implementation of the gearbox Sequential in the detection method of cooperation, dual CUSUM. The single detection node is not reliable, especially when nodes are subject to fading shadows due to barriers (hidden node problem). The results of the simulation indicate that the amount of channel transfers can be significantly reduced after using the intended use of the channels and the channel utilization is improved and that transfer of the blocking probability is also significantly reduced simultaneously. In addition, the results of additional simulations show that, apart from the activity of the PU, mobility node $\mathrm{CR}$ is also essential for the chain transfer and binding time available.

In [4], the author of this article suggests the method of admission control integrated slotted invitations for dynamic channel allocation to solve the problem. In the proposed method, the accepted user occurs at the beginning of a new slot; Therefore, the new OS coming between two slots must be first inserted into a queue until the next space arrives. By imposing a mandatory time-out limited so far to the new UB, the proposed method offers the possibility of activating the full OP permit using the available primary spectrum. An analytical framework using a 3D discrete time Markov chain has been developed to analyze the impact of the proposed method on the performance call level and SU packet level. The verification simulation results in the accuracy of the analysis and shows the effectiveness of the proposed method in terms of reducing the probability of blocking and drop, reducing packet time-up queuing and improving spectrum usage efficiency.

In [5], the author presents and analyzes the performance of the virtual reserve of collaborative knowledge networks. The virtual book is a strategy of maintaining narrative connections that aims to maximize the performance of the cognitive network with the full use of the spectrum. The performance evaluation shows a significant improvement not only in the probability of blocking and forced termination of the UB, but also the cognitive user rate.

In [6], the authors examine the allocation of resources in a cognitive network based on multiple orthogonal access databases (CR) that dynamically detects the spectrum of primary users (PU) and uses the available channels correctly. The objective is to allocate resources so that the performance of the RC network is maximized below the maximum interference PU and the power budget of the cognitive users (CU). Thus, the author formulated the problem of allocation of uplink resources in order to maximize the bit rate of the sum of the RC network (performance) under the transmission power to support the $\mathrm{CU}$ and PU to the maximum interference. In this article the author explains how to reformulate the subcarrier allocation problem in an equivalent problem defined in a conceptual system to an adaptive algorithm based on the least squares solution can be found.

In [7], the author examines the energy consumption problem of cognitive radio (CT) systems. The aim of this article is to maximize the energy efficiency of the CR system, taking into account practical constraints such as the CR system power budget, major user interference thresholds, minimum transmission requirements and user requirements. The net pro rata value.

In [13], the author studied the performance of network operators' data both on spectrum and power efficiency for LTEA networks.

In [14], the author presents the study on LTE networks of energy efficiency using the CA (carrier aggregation), in which the user equipment is capable of carrying different components.

\section{MOTIVATION}

In addition to equity in IEEE 802.22 wireless networks, it is another performance measure that relates to access to efficient dynamic spectrum (DSA) in a long-term evolution network (LTE) partners of the third generation cognitive (3GPP) architecture based on IEEE802.22. According to the composite of a number of IEEE802.22 standard service providers, wireless access is offered by a regional wireless area (WRAN) (PS) that share the total available spectrum 
using a spectrum manager (SM). The SM uses a flexible Dynamic Spectrum Access (DSA) policy to maximize capacity and quality of service (QoS) for users. Motivated by this CR (CRN) network concept, many articles have developed various forms of spectrum access strategies for allocating available network resources (bandwidth, transmission speed and transmission power). Most articles assume that non-core users greedy a general resource allocation policy. Recent methods have an efficient exchange between the transmission power, the bandwidth and the transmission speed of the LTE networks. This becomes the problem of current research in advance communication systems.

\section{PROPOSED SOLUTION}

To overcome the limitations of recent methods novel method proposed with goal of improving the spectrum and energy efficiency in LTE and LTE Advance Cellular Networks, this work distributed in three main parts:

- Quasiconvex optimization problem solving algorithm for optimal BS operation.

- MILP problem solving algorithms for near optimal solutions generation.

- Spectrum efficiency algorithm to further improve the capacity and energy efficiency.

Heuristic algorithms are designed to provide efficient computing solutions to the current optimization problem. The efficient energy method is designed to improve performance and energy efficiency. Figure 1 shows the overall design and performance parameters.

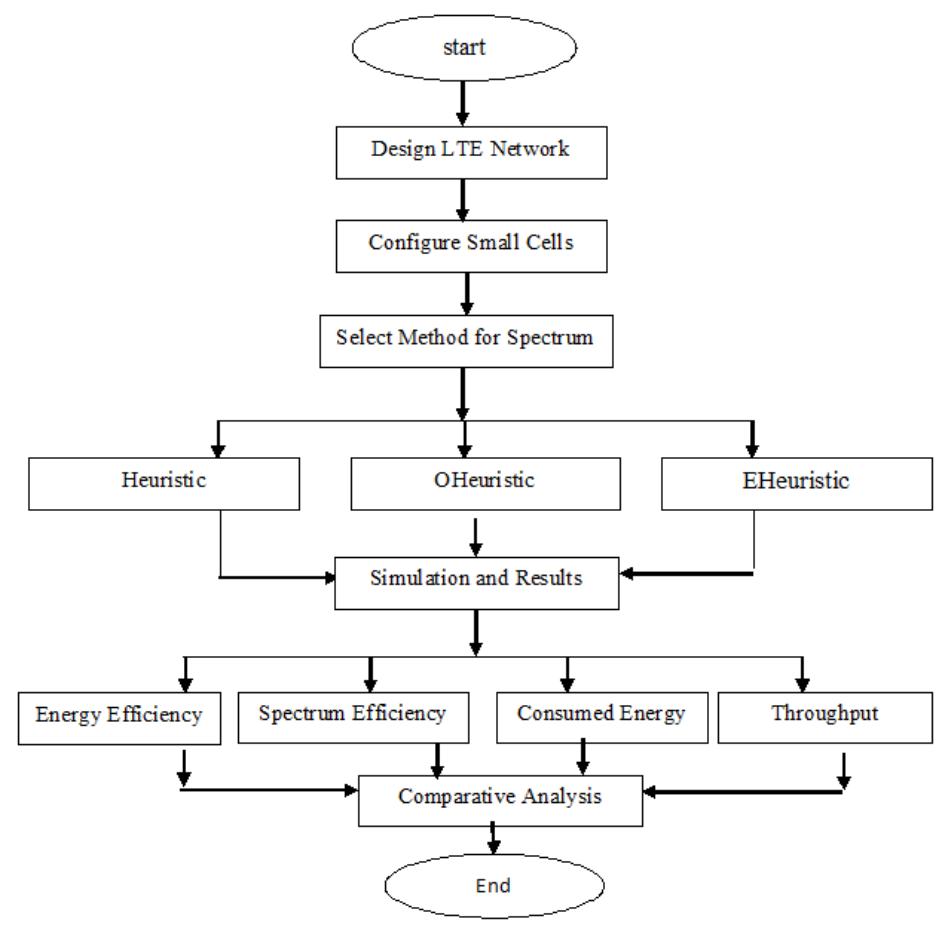

Figure 1: Design of Proposed Method in Class Diagram

The EHeuristic method below main algorithm of this work which is based on existing Heuristic and OHeuristic techniques.

\section{Algorithm: Energy-Spectrum Efficiency Algorithm}

Step 1: LTE-A Network Initialization

Step 2: Feasible point

Step 3: repeat

$$
\mathrm{x} \in \mathrm{R}^{2 \mathrm{KN}+1 \times 1}, \epsilon>0, \epsilon_{\mathrm{n}}>0, t=\mathrm{t}^{(0)}>0, \mu>1, \alpha \in\left(\frac{0,1}{2}\right), \beta \in(0,1) .
$$

Step 4: Apply Newton method

Step 5: Starting point $\mathrm{x}$, subject to $\mathrm{Bx}=1$

Step 6: repeat

Step 7: Compute 
$\Delta_{\mathrm{x}_{\mathrm{nt}}}$ and $\lambda^{2}=-\nabla_{\psi_{\mathrm{t}}}(\mathrm{x})^{\mathrm{T}} \Delta \mathrm{x}_{\mathrm{nt}}$

Step 8: Backtracking line search

Step 9: $\mathrm{s}=1$;

Step 10: while

Step 11: $\mathrm{s}=\beta_{\mathrm{s}}$

$$
\psi_{\mathbf{t}}\left(\mathrm{x}+\mathrm{s} \Delta \mathrm{x}_{\mathrm{nt}}\right)>\psi_{\mathbf{t}}(\mathrm{x})-\alpha \mathrm{s} \lambda^{2}
$$

Step 12: end while

Step 13: Update $\mathrm{x}=\mathrm{x}+s \Delta x_{n t} s \Delta \mathrm{x}_{\mathrm{nt}}$

Step 14: until $\lambda^{2} / 2 \leq \epsilon_{n \lambda^{2} / 2 \leq \epsilon_{\mathrm{n}}}$

Step 15: until $(3 \mathrm{KN}+\mathrm{K}+\mathrm{L}+3) / \mathrm{t}<\epsilon$

Step 16: return $\mathbf{x}$

Step 17: Energy-Spectrum Efficient Solution

Step 18: Stop

\section{RESULTS AND DISUCSSION}

The experimental analysis is carried out using a simulator known as NS2. We have designed several LTE networks with a variable number of users 50 to 300 with a simulation time of 30 seconds. Deployed 10 base stations per network. As shown in Figure 1, there are four measured performance measures are presented below

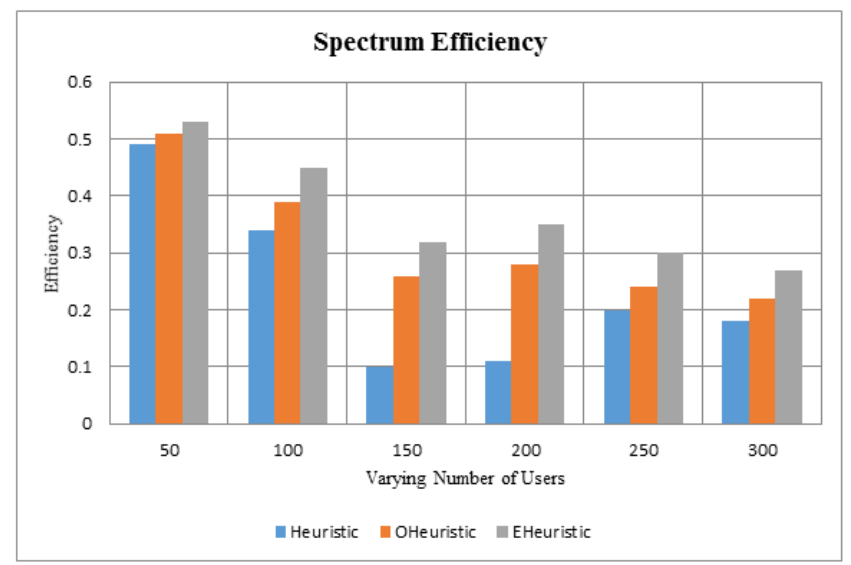

Figure 2: Performance Analysis of Spectrum Efficiency

Figure 2 is showing the performance for spectrum efficiency. The proposed method shows the improvement in spectrum efficiency as compared to existing methods such as Heuristic and OHeuristic for all types of networks from 500 to 300 users.

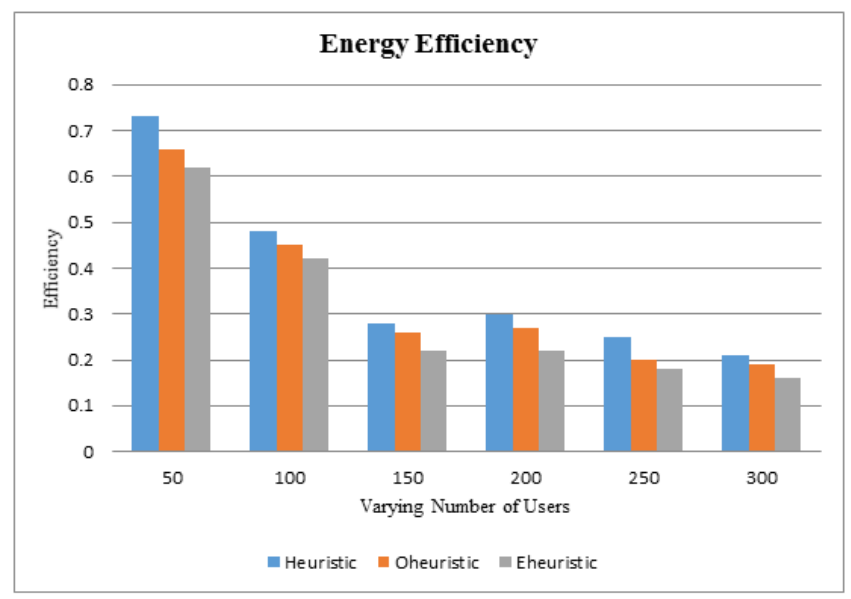

Figure 3: Performance Analysis of Energy Efficiency 


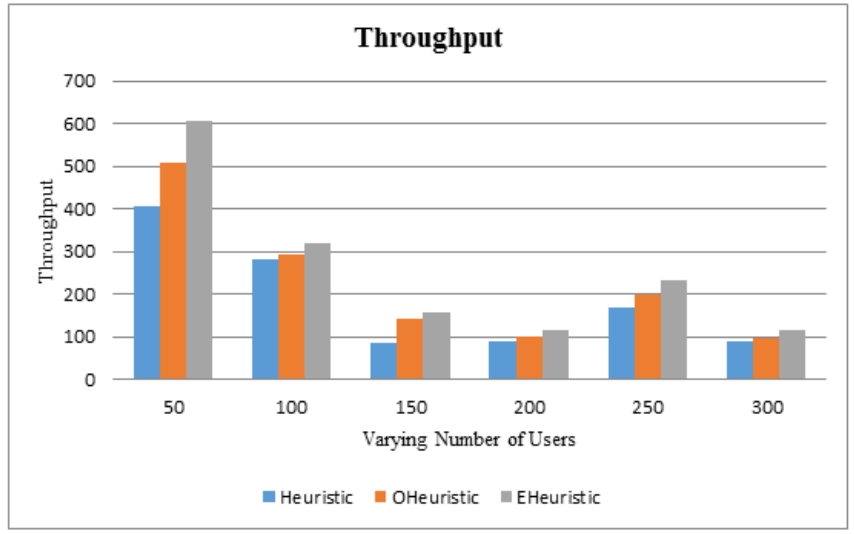

Figure 4: Performance Analysis of Throughput

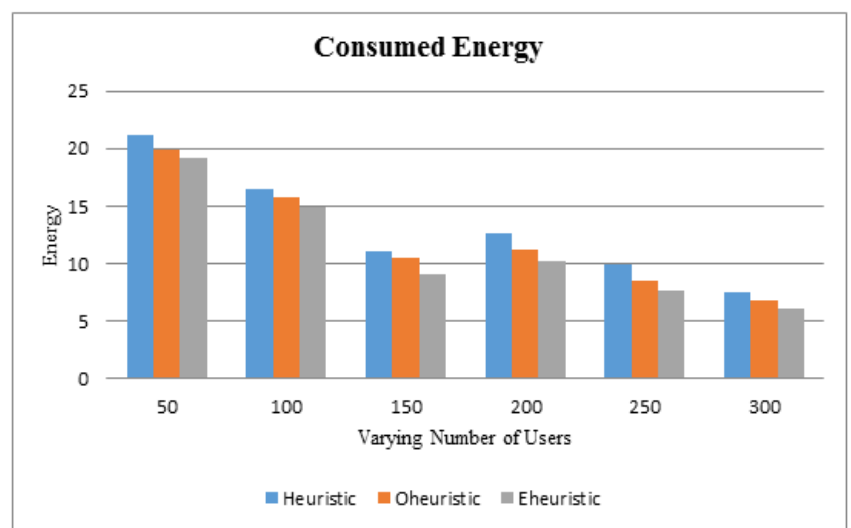

Figure 5: Performance Analysis of Consumed Energy

All results are showing that proposed approach shows the improvement in both spectrum and energy efficiency as compared to existing methods.

\section{VI.CONCLUSION AND FUTURE WORK}

We presented the method of spectrum efficiency and proposed hybrid with its design and simulation results in progress. The objective is to optimize the efficiency of spectrum efficiency, energy efficiency and LTE network throughput. In this report, the introduction, review of the literature is discussed. It is then presented to the system design, flowchart and work plan. The experimental study was carried out using ns 2 with different sizes of networks. The results show that the proposed approach improves energy efficiency by about $11 \%$. The efficiency of the spectrum makes it possible to improve by about $14 \%$ compared to the prior methods. For future work, we propose to work on the problems of mobility and traffic problems in LTE-A networks.

\section{REFERENCES}

[1] FCC. Et docket no. 03-322. "Notice of Proposed Rule Making and Order", Dec. 2003.

[2] Z.H. Hashmi, "Dynamic Resource Allocation for Cognitive Radio Systems", Master of Applied Science thesis, The University of British Columbia, June 2008.

[3] Yasmin Hassan and Sharjah, Sharjah, "Comparison of Linear and Polynomial Classifiers for Co-operative Cognitive Radio Networks", 2010.

[4] Ramachandra Budihal and Bharadwaj Desikan, "Co-operative Spectrum Sensing: Implementation and Benchmarking on ANR Cognitive Radio Testbed", 2012.

[5] Zhifeng Ni, Hangguan Shan*, Wei Shen t, Jian Wang, "Dynamic Channel Allocation-based Call Admission Control in Cognitive Radio Networks", 2013.

[6] Ayman T. Abdel-Hamid, Ahmed H. Zahran, "Improved Spectrum Mobility using Virtual Reservation in Collaborative Cognitive Radio Networks", 2013.

[7] Mahdi Raeis, Kamal Shahtalebi, Amir Reza Forouzan, "Computationally efficient adaptive algorithm for resource allocation in orthogonal frequency-division multiple-access-based cognitive radio networks”, IET Commun., 2015, Vol. 9, Iss. 12, pp. 1442-1449 1442 \& The Institution of Engineering and Technology 2015.

[8] Z. Niu, Y. Wu, J. Gong, and Z. Yang, “Cell zooming for cost-efficient green cellular networks,” Communications Magazine, IEEE, vol. 48, no. 11 , pp. $74-79$, November 2010. 
[9] J.-M. Kelif, M. Coupechoux, and F. Marache, "Limiting power transmission of green cellular networks: Impact on coverage and capa city," in Communications (ICC), 2010 IEEE International Conference on, May 2010, pp. 1 -6.

[10] S. Bhaumik, "Breathe to stay cool: Adjusting cell sizes to reduce energy consumption," Power, pp. 41-46, 2010.

[11] B. Badic, T. O'Farrrell, P. Loskot, and J. He, "Energy efficient radio access architectures for green radio: Large versus small cell size deployment," in Vehicular Technology Conference Fall (VTC 2009-Fall), 2009 IEEE 70th, September 2009, pp. 1 -5.

[12] G. Miao, N. Himayat, G. Li, and S. Talwar, "Low-complexity energy efficient scheduling for uplink OFDMA," IEEE Trans. Commun., vol. 60, no. 1, pp. 112-120, Jan. 2012

[13] Chan-Ching Hsu and J. Morris Chang, "Spectrum-Energy Efficiency Optimization for Downlink LTE-A for Heterogeneous Networks", IEEE Transactions on Mobile Computing, 2016.

[14] Soheil Rostami, Kamran Arshad, "Energy-Efficient Resource Allocation for LTE-A Networks", IEEE Communications Letters, 2016.

\section{BIOGRAPHIES}

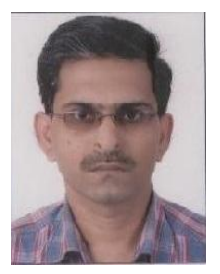

Y. D. Chincholkar received B.E. degree in Electronics and Telecommunication Engineering from Shri Sant Gajanan Maharaj College of Engineering, Shegaon, Amravati University, Amravati, Maharashtra, India, in 1997. In 2000 and 2008, he received the M.B.A degree in Marketing Management from Shri Sant Gajanan Maharaj College of Engineering, Shegaon, Amravati University and Master of Engineering from Vivekanand Education Society's Institute of Technology, Chembur, Mumbai, Mumbai University, Mumbai, Maharashtra, India. He is currently a research scholar in the Department of Electronics and Telecommunication of Sinhgad College of Engineering, Pune, India. His research interest includes signal processing and communication.

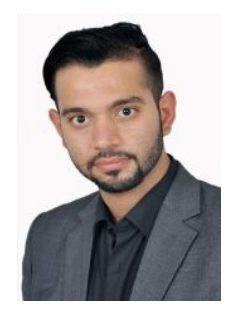

Mohammed Luay Abdulmunem received B.E. degree in Electronics and Communication Engineering from Al-Mamon University college, Iraq, Baghdad in 2013, He is currently research M.E degree in SCOE college, Savitribai Phule Pune University, interest is in the research areas of wireless communication network, LTE and LTE-A network, Optical communication network [EPON], modulation technic, GSM network optimization . 